\title{
Observation of Zonal Flow Type Oscillation in Linear Cylindrical ECR Plasmas
}

\author{
Kunihiro KAMATAKI, Sanae -I. ITOH ${ }^{1)}$, Yoshihiko NAGASHIMA ${ }^{2)}$, Shigeru INAGAKI ${ }^{1)}$, \\ Shunjiro SHINOHARA, Masatoshi YAGI ${ }^{1)}$, Takuma YAMADA ${ }^{2)}$, Yoshinobu KAWAI, \\ Akihide FUJISAWA ${ }^{3)}$ and Kimitaka ITOH $^{3)}$ \\ Interdisciplinary Graduate School of Engineering Science, Kyushu University, Kasuga, Fukuoka 816-8580, Japan \\ ${ }^{1)}$ Research Institute for Applied Mechanics, Kyushu University, Kasuga, Fukuoka 816-8580, Japan \\ ${ }^{2)}$ Graduate School of Frontier Sciences, The University of Tokyo, Kashiwa, Chiba 277-8561, Japan \\ ${ }^{3)}$ National Institute for Fusion Science, Toki, Gifu 509-5292, Japan
}

(Received 3 June 2008 / Accepted 12 July 2008)

\begin{abstract}
Zonal flow type oscillation, which is driven by a small modulation of the electron cyclotron resonance (ECR) power in a low-frequency band $(<0.1 \mathrm{kHz})$, and its impact on drift wave turbulence are observed in linear cylindrical ECR plasmas by simultaneous spatiotemporal measurements with a multi-ring probe array. It is found that a potential low-frequency $(<0.1 \mathrm{kHz})$ oscillation can have a zonal flow type structure. Bicoherence analysis reveals that this zonal flow type oscillation has a nonlinear interaction with the drift mode. These results indicate that an electric field with zonal flow, which is excited externally, can modulate the drift mode amplitude, similar to spontaneous zonal flow.
\end{abstract}

(C) 2008 The Japan Society of Plasma Science and Nuclear Fusion Research

Keywords: zonal flow type oscillation, drift wave turbulence, ECR modulation, linear cylindrical ECR plasma

DOI: $10.1585 /$ pfr.3.047

The regulation or suppression of plasma turbulence by a zonal flow is considered to be a control knob for plasma confinement. It was theoretically and numerically demonstrated that zonal flow can be generated by the nonlinear interaction of the plasma turbulence, and regulates the turbulence itself [1-3]. Zonal flows have anisotropic potential structures, with toroidal and poloidal symmetries (poloidal and parallel mode numbers $m=0$ and $n=0$, respectively), and they are radially localized (radial wave number $k_{\mathrm{r}} \neq 0$ ). Zonal flows modulate the turbulence through the shear suppression mechanism. Fujisawa et al. confirmed the presence of zonal flows in a magnetically confined toroidal plasma device, compact helical system (CHS), using dual heavy ion beam probes $[4,5]$. Several studies on zonal flows have been reported using various diagnostics [6-8]. Recently, Nagashima et al. identified the parametric-modulational instability of the drift wavezonal flow system in a linear cylindrical Large Mirror Device (LMD), using a Reynolds stress probe [9-11]. Komori et al., reported the influence of $\vec{E} \times \vec{B}$ sheared flow in a coherent mode using a biased separated end plate, which controls the mean radial electric field [12]. However, the interaction of externally induced time varying $\vec{E} \times \vec{B}$ shear flow with the drift mode has not been reported. In this letter, we present experimental observations of the impact of an oscillation driven by a small modulation of ECR power, which has the zonal flow structure (called a zonal flow type

author'se-mail: kamataki@riam.kyushu-u.ac.jp oscillation in this letter), on the drift wave.

Experiments are performed in the region of lower ionneutral collision frequency $v_{\text {in }} / \Omega_{\mathrm{i}} \sim 0.4$ (filling gas pressure $p_{(\mathrm{Ar})}=0.6 \times 10^{-3}$ Torr), where the drift mode is strongly excited in a linear cylindrical electron cyclotron resonance (ECR) plasma [13]. Here, $v_{\text {in }}$ and $\Omega_{\mathrm{i}}$ indicate the ion-neutral collision frequency and the ion cyclotron frequency, respectively. A multi-ring probe array [14] is used to observe the radial structure of the radial electric field oscillation. The magnetic coil assembly consisted of eight coils (axial width of $6.4 \mathrm{~cm}$, and inner and outer diameters of 60 and $88 \mathrm{~cm}$, respectively) forming a mirror magnetic field, with a mirror ratio of 1.5. A microwave with a frequency of $2.45 \mathrm{GHz}$ and output power of $P_{\mu} \leq$ $1 \mathrm{~kW}$ is launched into a vacuum chamber (with a $40 \mathrm{~cm}$ inner diameter and $120 \mathrm{~cm}$ axial length $120 \mathrm{~cm}$ ) in a circular $\mathrm{TE}_{11}$ mode through a coaxial waveguide converter $(10 \mathrm{~cm}$ inner diameter and $21 \mathrm{~cm}$ axial length), which is mounted at the end of the vacuum chamber. The total axial length of the plasma including the converter is about $140 \mathrm{~cm}$ and the plasma radius is $5 \mathrm{~cm}$. The ECR discharge is performed continuously for $2 \mathrm{~s}$. The spatiotemporal structures of the ion saturation current $I_{\text {is }}$ and floating potential $V_{\mathrm{f}}$ were measured simultaneously using a multi-ring probe array that has eight stainless steel rings $(3 \mathrm{~mm}$ outer diameter, $2.5 \mathrm{~mm}$ inner diameter, and radial length of $1.2 \mathrm{~mm}$ with a radial central separation of $10 \mathrm{~mm}$ ) positioned in the radial direction. The averaged plasma parameters (the elec- 


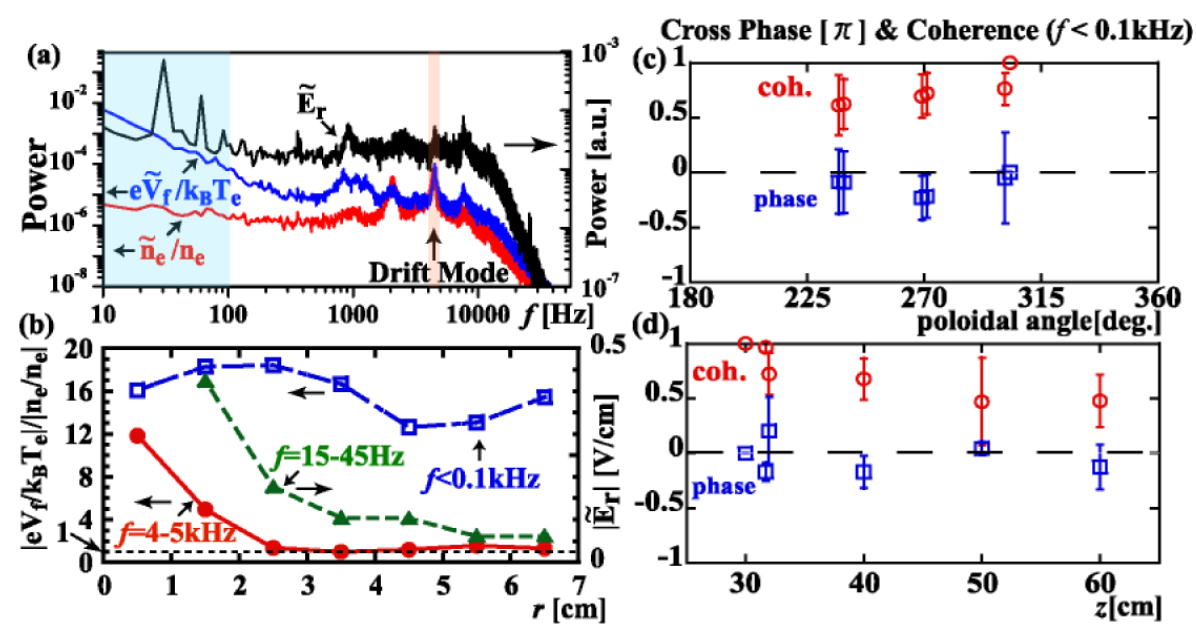

Fig. 1 (a) Auto-power spectra of normalized density $\tilde{n}_{\mathrm{e}} / n_{\mathrm{e}}\left(\right.$ red), normalized potential $e \tilde{V}_{\mathrm{f}} / k_{\mathrm{B}} T_{\mathrm{e}}$ (blue) and radial electric field $\tilde{E}_{\mathrm{r}}$ (black) at $r=4.5 \mathrm{~cm}$. The signal magnitude without plasma in the low-frequency band (60 Hz component is dominant) are at least seven orders smaller than the above mentioned signals. (b) Radial profiles of $\left|e \tilde{V}_{\mathrm{f}} / k_{\mathrm{B}} T_{\mathrm{e}}\right| /\left|\tilde{n}_{\mathrm{e}} / n_{\mathrm{e}}\right|$ at $f=4-5 \mathrm{kHz}$ (red) and $f<0.1 \mathrm{kHz}$ (blue) and amplitude of $\left|\tilde{E}_{\mathrm{r}}(f=15-45 \mathrm{~Hz})\right|$ (green). The cross phase (divided by $\pi$ ) and cross coherence between floating potentials $(f<0.1 \mathrm{kHz})$ at (c) poloidal (at $z=30 \mathrm{~cm})$ and (d) $z$ directions (at $\theta=270 \mathrm{deg}$.) in the same radial direction $r=4.5 \mathrm{~cm}$.

tron density $n_{\mathrm{e}}$ and temperature $T_{\mathrm{e}}$ ) are obtained from the current-voltage $(I-V)$ characteristics of the single Langmuir probe. Experiments were performed in the region where the drift mode is dominant under the following conditions: the magnetic field strength $B=685$ Gauss, $P_{\mu}=$ $300 \mathrm{~W}, p_{(\mathrm{Ar})}=0.6 \times 10^{-3}$ Torr, $n_{\mathrm{e}}=2 \times 10^{11} \mathrm{~cm}^{-3}$, and $T_{\mathrm{e}}$ $=2 \mathrm{eV}$.

Figure 1 (a) shows the auto-power spectra of the normalized density $\tilde{n}_{\mathrm{e}} / n_{\mathrm{e}}$ (red), normalized potential $e \tilde{V}_{\mathrm{f}} / k_{\mathrm{B}} T_{\mathrm{e}}$ (blue) ( $k_{\mathrm{B}}$ is the Boltzmann constant) and radial electric field fluctuation $\tilde{E}_{\mathrm{r}}$ (black) at $r=4.5 \mathrm{~cm}$, where $\tilde{A}(t)=$ $A(t)-<A(t)>\left(<>\right.$ : the temporal average) and $\tilde{E}_{\mathrm{r}}(r, t)=$ $-\left(\tilde{V}_{\mathrm{f}}(r+\Delta r, t)-\tilde{V}_{\mathrm{f}}(r, t)\right) / \Delta r, \Delta r=1 \mathrm{~cm}$ is calculated from two adjacent positions of the multi-ring probe array with a spatial distance of $1 \mathrm{~cm}$. The frequency resolution is $6.1 \mathrm{~Hz}$ and 100 ensembles are averaged to reduce the statistical error. It is found that the power spectra of $\tilde{E}_{\mathrm{r}}$ have peaks in the low-frequency band $(f<0.1 \mathrm{kHz})$ and the maximum peak is observed at $f=30 \mathrm{~Hz}$. The spectrum of $e \tilde{V}_{\mathrm{f}} / k_{\mathrm{B}} T_{\mathrm{e}}$ contains the overall potential fluctuations which will cancel out $\tilde{E}_{\mathrm{r}}$ estimation, and thus is broad in the lowfrequency $\left(f<0.1 \mathrm{kHz}\right.$ ) band. In fact, $e \tilde{V}_{\mathrm{f}} / k_{\mathrm{B}} T_{\mathrm{e}}$ has a strong coherence $(\sim 0.7)$ with $\tilde{E}_{\mathrm{r}}$ at $f=30 \mathrm{~Hz}$ (not shown). Thus, we focus on modes at $f=30 \mathrm{~Hz}$. The radial profiles of $\left|\tilde{E}_{\mathrm{r}}\right|$ at $f=15-45 \mathrm{~Hz}$ (green) are shown in Fig. 1 (b). The ratio of the normalized potential to density fluctuation levels, i.e., $\left|e \tilde{V}_{\mathrm{f}} / k_{\mathrm{B}} T_{\mathrm{e}}\right| /\left|\tilde{n}_{\mathrm{e}} / n_{\mathrm{e}}\right|$ is useful in identifying the drift modes. The radial profile of $\left|e \tilde{V}_{\mathrm{f}} / k_{\mathrm{B}} T_{\mathrm{e}}\right| /\left|\tilde{n}_{\mathrm{e}} / n_{\mathrm{e}}\right|$ at the drift frequency $(4-5 \mathrm{kHz})$ (red) is also shown in Fig. 1 (b). This indicates a drift mode feature i.e. $\left|e \tilde{V}_{\mathrm{f}} / k_{\mathrm{B}} T_{\mathrm{e}}\right| /\left|\tilde{n}_{\mathrm{e}} / n_{\mathrm{e}}\right| \sim 1$ at $r \sim 3-5 \mathrm{~cm}$. In the low-frequency band $(f<0.1 \mathrm{kHz})$, the magnitude of the normalized potential fluctuation is one order larger than the density at all radial positions, which is one of the features of zonal flows. The radial profile of $\left|\tilde{E}_{\mathrm{r}}\right|$ at $f=15-45 \mathrm{~Hz}$ peaks at $r=1.5 \mathrm{~cm}$, and decreases toward the outer radial region. Figures 1 (c) and (d) show the cross phases and cross coherences of $\tilde{V}_{\mathrm{f}}$ between two separated positions in the low-frequency band $(f<0.1 \mathrm{kHz})$ (here, the significant level of this cross coherence is 0.01 .). The reference probe is located at $(r, \theta, z)=\left(4.5 \mathrm{~cm}, 303^{\circ}\right.$, $30 \mathrm{~cm})$. The cylindrical coordinate system in which axes $(r, \theta, z)$ satisfy the right-hand rule is employed. Here, $z$ is measured from the coaxial waveguide converter. The $\theta$ coordinate is defined such that the vertical (downward) direction from the center of the device has a $\theta$ of 270 degrees [13]. The cross phases in both azimuthal and axial directions are regarded as near zero within the present error bars, and show the azimuthal and axial symmetric structures of $(m, n)=(0,0)$, similar to a zonal flow structure. Here, $m$ and $n$ are the azimuthal and the axial mode numbers, respectively. In addition, the azimuthal mode number $m$ of $\tilde{E}_{\mathrm{r}}$ at $f=30 \mathrm{~Hz}$ is identified as 0 by two point $\tilde{E}_{\mathrm{r}}$ measurements with a finite azimuthal angle (about 60 degrees) (not shown). This result supports that the idea that $\tilde{E}_{\mathrm{r}}$ at $f=30 \mathrm{~Hz}$ has an axial symmetry $(m=0)$. The $\tilde{V}_{\mathrm{f}}$ at $f<0.1 \mathrm{kHz}$ is in phase with a strong coherence $(>0.5)$ in an axial magnetic field line direction as shown in Fig. 1 (d) and has strong coherence $(\sim 0.7)$ with $\tilde{E}_{\text {r }}$ at $f=30 \mathrm{~Hz}$ in the same radial position in this experiment. Thus, we assumed that $\tilde{E}_{\mathrm{r}}$ at $f=30 \mathrm{~Hz}$ also has an axial symmetric structure of $n=0$ like $\tilde{V}_{\mathrm{f}}$ at $\left.f<0.1 \mathrm{kHz}\right)$. The mode structure of $\tilde{E}_{\mathrm{r}}$ at $f=30 \mathrm{~Hz}$ is considered to be $(m, n)=(0$, $0)$.

The spatiotemporal characteristics of the radial electric field (at low frequency, $f<0.1 \mathrm{kHz}$ ) are given by calculating the two-points correlation function, de- 

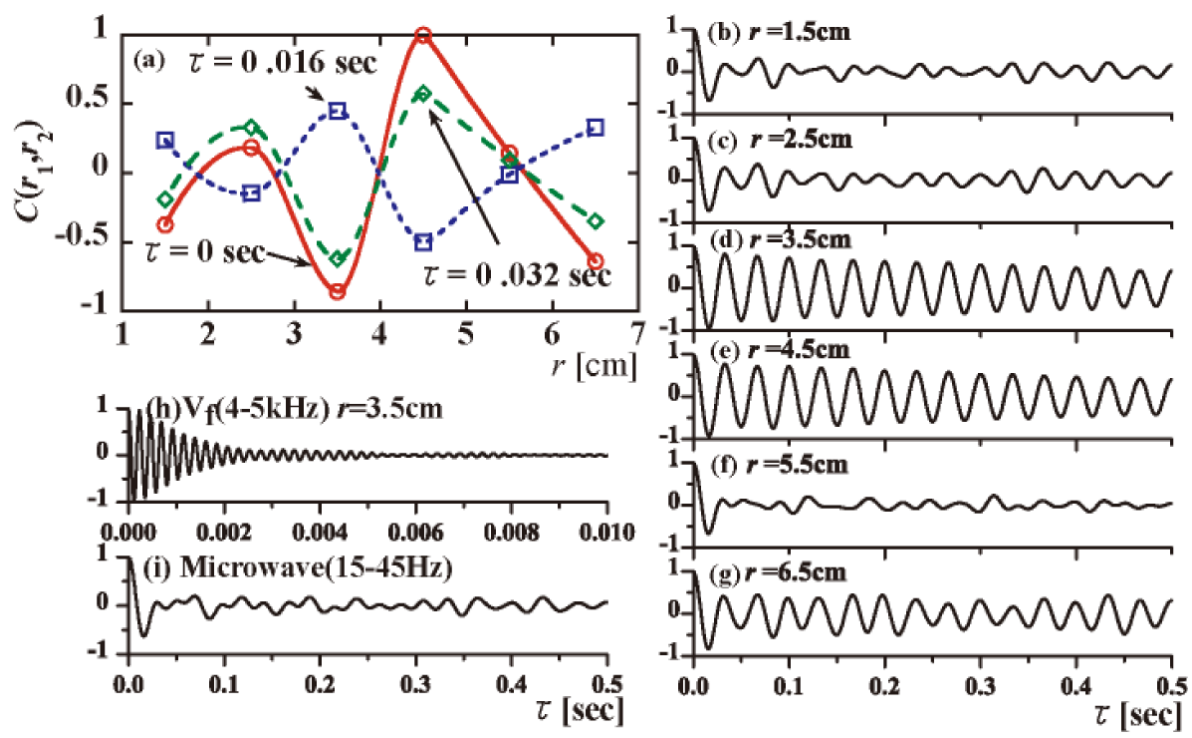

Fig. 2 (a) Correlation functions between the radial electric fields $(f<0.1 \mathrm{kHz})$ at different radial positions around $r=4.5 \mathrm{~cm}$ with three time delays; $\tau=0,0.016$ and $0.032 \mathrm{~s}$. (b) - (g) Auto correlation functions of $\tilde{E}_{\mathrm{r}}(f=15-45 \mathrm{~Hz})$ at $r=1.5-6.5 \mathrm{~cm}$, (h) of $\tilde{V}_{\mathrm{f}}(f=4$ $5 \mathrm{kHz})$ at $r=3.5 \mathrm{~cm}$, and (i) of the input microwave $(f=15-45 \mathrm{~Hz})$.

fined as $C\left(r_{1}, r_{2} ; \tau\right)=\left\langle\tilde{E}_{\mathrm{r}}\left(r_{1} ; t\right) \cdot \tilde{E}_{\mathrm{r}}\left(r_{2} ; t+\tau\right)\right\rangle /$ $\sqrt{\left\langle\tilde{E}_{\mathrm{r}}\left(r_{1} ; t\right)^{2}\right\rangle \cdot\left\langle\tilde{E}_{\mathrm{r}}\left(r_{2} ; t\right)^{2}\right\rangle}$, where $\langle\psi\rangle=(1 / T) \times \int_{-T / 2}^{T / 2} \psi \mathrm{d} t$. Here, $T$ is $1 \mathrm{~s}$ and the number of ensembles is 10 . Cross correlation of $\tilde{E}_{\mathrm{r}}$ in the low-frequency band $(f<0.1 \mathrm{kHz})$ is obtained by shifting the probe tip position $r_{2}$, shot-byshot, while fixing the other at $r_{1}=4.5 \mathrm{~cm}$. Figure 2 (a) shows $C\left(r_{1}, r_{2} ; \tau\right)$ at three points in times $(\tau=0,0.016$, and $0.032 \mathrm{~s})$. These show the simultaneous radial structure in the low-frequency band $(f<0.1 \mathrm{kHz}$ ), which seems to be a quasi-sinusoidal radial structure with a radial wavelength of $\lambda_{\mathrm{r}} \sim 2.5 \mathrm{~cm}$. This wavelength of zonal flow type oscillation is approximately eight times larger than the ion Larmor radius (micro scale) and less than one-half of the plasma radius (macro scale). A characteristic meso scale is given by [1] $\sqrt{\rho_{\mathrm{s}} a}=1.2 \mathrm{~cm}$, which is in a factor of $\sim 2$ of the observed one. This quasi-sinusoidal radial structure has the cycle with $f=30 \mathrm{~Hz}$. (As shown in Fig. 1 (a), The autopower spectra of $\tilde{E}_{\mathrm{r}}$ also have peaks at $f=30 \mathrm{~Hz}$.) Here, we consider the main component $(f=30 \mathrm{~Hz})$ of $\tilde{E}_{\mathrm{r}}$ in the radial direction. The auto-correlation functions $C(r ; \tau)$ of $\tilde{E}_{\mathrm{r}}(f=15-45 \mathrm{~Hz})(T=1 \mathrm{~s}$ and 10 ensembles. $)$ at $r=1.5$ $-6.5 \mathrm{~cm}$ (shown in Figs $2(\mathrm{~b})-(\mathrm{g})$ ) indicate that the zonal flow type oscillations are coherent and have a long lifetime (>0.5 s). In particular, at $r=3.5$ and $4.5 \mathrm{~cm}$, the lifetime is much longer than that of the drift mode $(\sim 2 \mathrm{~ms})$ (shown in Fig. $2(\mathrm{~h})$ ). Thus, observed zonal flow type oscillation is not considered to be produced by the secondary (modulational) instability of drift waves. In this letter, the oscillations produced by the modulational instability of drift waves and by the influence of external sources are defined as the spontaneous and the externally driven zonal flow oscillations, respectively. Next, we studied the origin of this zonal flow type oscillation. As shown in Fig. 1 (b), the maximum amplitude of $\left|\tilde{E}_{\mathrm{r}}(f=15-45 \mathrm{~Hz})\right|$ at $r=1.5 \mathrm{~cm}$ indicates that ECR power is mainly absorbed in the central region. In addition, the auto correlation function of the input microwave ( $f=15-45 \mathrm{~Hz}$ ) (shown in Fig. 2 (i)) is similar to that of the $\tilde{E}_{\mathrm{r}}(f=15-45 \mathrm{~Hz})$ at $r=1.5 \mathrm{~cm}$ (shown in Fig. 2(b)). We then study the relation between the modulation of the ECR heating power and the observed oscillation. Figure 3 (a) shows auto-power spectra of $\tilde{E}_{\mathrm{r}}$ at $r=1.5-4.5 \mathrm{~cm}$ at $f<0.1 \mathrm{kHz}$. Though the spectrum of $\tilde{E}_{\mathrm{r}}$ is broad near the plasma center $(r=1.5 \mathrm{~cm})$, it is coherent at outer radial positions. To understand the causal relationships between $\tilde{E}_{\mathrm{r}}(f=15-45 \mathrm{~Hz})$ and the input microwave ( $f=15-45 \mathrm{~Hz}$ ), the cross correlation function between their envelopes is calculated in the phase where the amplitude of the input microwave changes (shown in Figs. 3 (b) at $r=1.5 \mathrm{~cm}$ and (c) at $r=2.5 \mathrm{~cm}$ ). Here, $T=0.3 \mathrm{sec}$, there are 10 ensembles, and the reference of this analysis is the input microwave ( $f=15-45 \mathrm{~Hz}$ ). Peaks of the cross correlation functions at $r=1.5$ and $2.5 \mathrm{~cm}$ are large $(>0.7)$, thus the amplitudes of $\tilde{E}_{\mathrm{r}}$ and the microwave have a strong correlation. The time-to-peak of the cross correlation (the time delay) $\tau$ at $r=1.5 \mathrm{~cm}$ is almost 0 and $\tau=-0.007 \mathrm{~s}$ at $r=2.5 \mathrm{~cm}$. The negative $\tau$ means that the amplitudes of $\tilde{E}_{\mathrm{r}}(f=15-45 \mathrm{~Hz})$ is altered by changes in the input microwave oscillation $(f=15-45 \mathrm{~Hz})$. Thus the $\tilde{E}_{\mathrm{r}}$ is considered to be generated by the input microwave oscillation and its generation mechanisms have a time scale of $0.007 \mathrm{~s}$. The observed time delay of $0.007 \mathrm{~s}$ is shorter than the lifetimes of $\tilde{E}_{\mathrm{r}}(f=15-45 \mathrm{~Hz})(>0.03 \mathrm{~s})$ at all radial positions (shown in Figs. $2(\mathrm{~b})-(\mathrm{g})$ ). The $\tilde{E}_{\mathrm{r}}$ oscillation with a zonal flow structure is therefore induced from a small perturbation of input microwave power. It has been reported that 

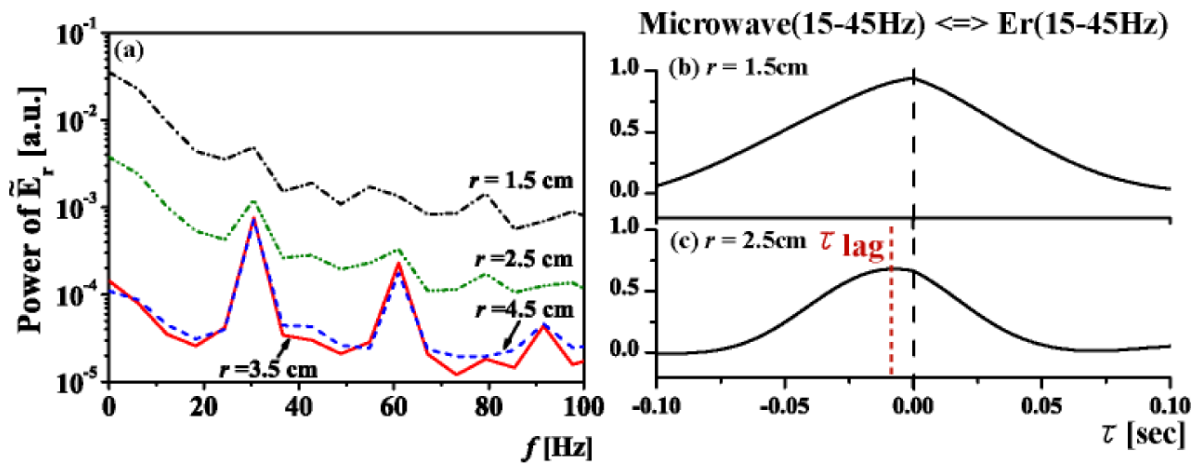

Fig. 3 (a) Auto-power spectra of $\tilde{E}_{\mathrm{r}}$ at $r=1.5-4.5 \mathrm{~cm}$ at $f<0.1 \mathrm{kHz}$ and, (b) and (c) the cross correlation functions between envelopes of the input microwave $(f=15-45 \mathrm{~Hz})$ and $\tilde{E}_{\mathrm{r}}(f=15-45 \mathrm{~Hz})$ at $r=1.5$ and $2.5 \mathrm{~cm}$.

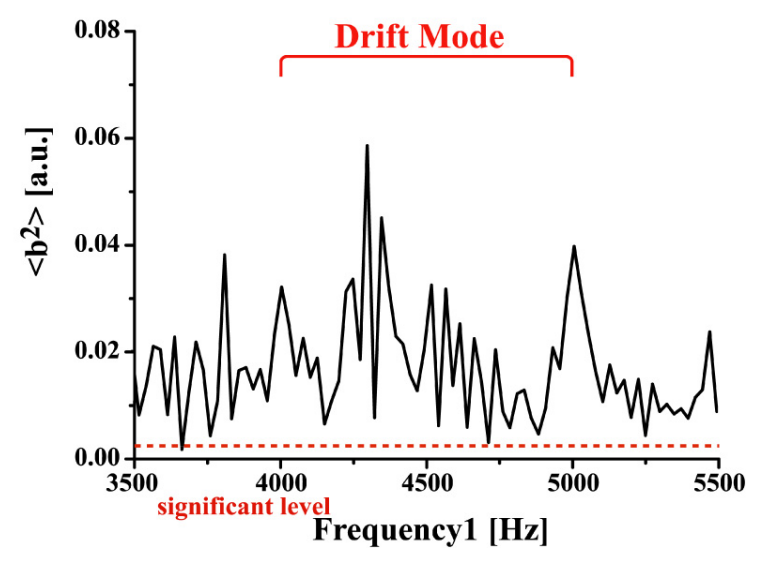

Fig. 4 Auto-bicoherence of the floating potential fluctuation at $f_{3}<0.1 \mathrm{kHz}$ (frequency of zonal flow type oscillation). The horizontal axis shows $f_{1}$. The significance level is $\sim$ 0.002 .

the ECR power modulation can excite electric field oscillations [15]. The zonal flow type electric fields $\tilde{E}_{\mathrm{r}}$ at $r=$ 3.5 and $4.5 \mathrm{~cm}$ have coherent mode at $f=30 \mathrm{~Hz}$ and its higher harmonics. The reason why $\tilde{E}_{\mathrm{r}}$ at $r=3.5$ and $4.5 \mathrm{~cm}$ has especially long autocorrelation times at $f=30 \mathrm{~Hz}$ is unknown. This issue corresponds to the mechanism by which $\tilde{E}_{\mathrm{r}}$ is excited by external power modulation, and is left for the future work.

This externally induced zonal flow type oscillation regulates the ambient drift waves. Figure 4 shows an auto-bicoherence plot of the floating potential fluctuations $\left\langle b^{2}\right\rangle=\int_{f_{3}<0.1 \mathrm{kHz}}\left\langle\tilde{V}_{\mathrm{f}}\left(f_{1}\right) \tilde{V}_{\mathrm{f}}\left(f_{2}=f_{3}-f_{1}\right) \tilde{V}_{\mathrm{f}}\left(f_{3}\right)\right\rangle \mathrm{d} f$. Here, the frequency resolution is $24.4 \mathrm{~Hz}$ and the significance level is 0.002 . Auto-bicoherence analysis indicates that the nonlinear interaction between the drift mode and the zonal type oscillation is significant, which is similar to the observed spontaneous zonal flow in LMD [10].

In conclusion, we have observed zonal flow type oscillation in a linear cylindrical ECR plasma using simultaneous spatiotemporal measurements with a multi-ring probe array. It was found that low-frequency $(<0.1 \mathrm{kHz})$ radial electric field oscillation, which is induced from microwave power modulation, has a zonal flow structure. Bicoherence analysis revealed that externally driven zonal flow type oscillation has a nonlinear interaction with the drift mode. These results indicate that radial electric field oscillations with a zonal flow type structure excited by an external source can modulate and control the drift mode amplitude.

We would like to acknowledge useful discussions with Dr. A. Fukuyama, Dr. N. Kasuya, and Dr. S. Nishimura. This work is partly supported by a Grant-inAid for Specially-Promoted Research of MEXT of Japan (16002005), by Research Fellowships of the Japan Society for the Promotion of Science for Young Scientists, and by a collaborative program between the Research Institute for Applied Mechanics of Kyushu University and The National Institute for Fusion Science (NIFS) (NIFS07KOAP017).

[1] P.H. Diamond, S.-I. Itoh, K. Itoh and T.S. Hahm, Plasma Phys. Control. Fusion 47, R35 (2005).

[2] A. Hasegawa and M. Wakatani, Phys. Rev. Lett. 59, 1581 (1987).

[3] Z. Lin, T.S. Hahm, W.W. Lee, W.M. Tang and R.B. White, Science 281, 1835 (1998)

[4] A. Fujisawa, K. Itoh, H. Iguchi, K. Matsuka, S. Okamura, A. Shimizu, T. Minami, Y. Yoshimura, K. Nagaoka, C. Takahashi, M. Kojima, H. Nakano, S. Ohsima, S, Nishimura, M. Isobe, C. Suzuki, T. Akiyama, K. Ida, K. Toi, S.-I. Itoh and P.H. Diamond, Phys. Rev. Lett. 93, 165002 (2004)

[5] A. Fujisawa, K. Itoh, A. Shimizu, H. Nakano, S. Ohsima, H. Iguchi, K. Matsuoka, S. Okamura, T. Minami, Y. Yoshinuma, K. Nagaoka, K. Ida, K. Toi, C. Takahashi, M. Kojima, S, Nishimura, M. Isobe, C. Suzuki, T. Akiyama, Y. Nagashima, S.-I. Ioh and P.H. Diamond, Phys. Rev. Lett. 98, 165001 (2007).

[6] D.K. Gupta, R.J. Fonck, G.R. McKee, D.J. Schlossberg and M.W. Shafer, Phys. Rev. Lett. 97, 125002 (2006).

[7] Y. Hamada, A. Nishizawa, T. Ido, T. Watari, M. Kojima, Y. Kawasumi, K. Narihara, K. Toi and JIPPT-IIU Group, Nucl. Fusion 45, 81 (2005).

[8] C. Holland, J.H. Yu, A. James, D. Nishijima, M. Shimada, N. Taheri and G.R. Tynan, Phys. Rev. Lett. 96, 195002 (2006). 
[9] Y. Nagashima, S.-I. Itoh, S. Shinohara, M. Fukao, A. Fujisawa, K. Terasaka, Y. Kawai, G.R. Tynan, P.H. Diamond, M. Yagi, S. Inagaki, T. Yamada and K. Itoh, submitted to Phys. Rev. Lett.

[10] Y. Nagashima, S.-I. Itoh, S. Shinohara, M. Fukao, A. Fujisawa, K. Terasaka, Y. Kawai, N. Kasuya, G.R. Tynan, P.H. Diamond, M. Yagi, S. Inagaki, T. Yamada and K. Itoh, submitted to J. Phys. Soc. Jpn.

[11] Y. Nagashima, S.-I. Itoh, S. Shinohara, M. Fukao, A. Fujisawa, T. Nishizima, K. Terasaka, M. Kawaguchi, Y. Kawai, G.R. Tynan, P.H. Diamond, M. Yagi, S. Inagaki, T. Yamada, T. Maruta, K. Kamataki and K. Itoh, Bulletin of 49th Meeting of Division of Plasma Physics of American
Physics Society, YP8.00073 (2007).

[12] A. Komori, K. Watanabe and Y. Kawai, Phys. Fluids 31, 210 (1988).

[13] K. Kamataki, Y. Nagashima, S. Shinohara, Y. Kawai, M. Yagi, K. Itoh and S.-I. Itoh, J. Phys. Soc. Jpn. 76, 054501 (2007).

[14] K. Kamataki, S.-I. Itoh, Y. Nagashima, S. Inagaki, S. Shinohara, M. Yagi, T. Yamada, Y. Kawai, A. Fujisawa and K. Itoh, Plasma Phys. Control. Fusion 50, 035011 (2008).

[15] H. Tsuchiya, S.-I. Itoh, A. Fujisawa, K. Kamataki, S. Shinohara, M. Yagi, Y. Kawai, A, Komori and K. Itoh, Plasma Phys. Control. Fusion 50, 055005 (2008). 